\title{
Modelling of an Underground Waste Disposal Site by Upscaling and Simulation with Domain Decomposition Method
}

\author{
I. Boursier ${ }^{1}$, A. Bourgeat ${ }^{1}$, and D. Tromeur-Dervout ${ }^{1}$ \\ Modelling and Scientific Computing Laboratory, University Lyon 1, France
}

\begin{abstract}
Summary. We derive an upscaled but accurate 2D model of the global behavior of an underground radioactive waste disposal. This kind of computation occurs in safety assessment process. Asymptotic development of the solution leads to solve terms of order 1 on more regular and steady-state auxiliary problems. Neumann-Dirichlet domain decomposition methods, with non matching spectral grids, are performed to solve those auxiliary problems. Fourier and Chebychev polynomials approximation of the solution are used depending on boundary conditions implemented on subdomains. Since spectral representation of the solution or its derivatives allows accurate mappings between the interfaces of the different grids, we speed up the convergence of the Neumann-Dirichlet method by the Aitken acceleration which is sensitive to the accuracy of the representation of the iterate solution on the artificial interfaces. In order to enforce regularity for the spectral approximation, some regular extensions and filtering techniques on the artificial interfaces for the right hand side of the problem and the iterate solution are implemented.
\end{abstract}

\section{Field decomposition method applied to an underground waste disposal}

The disposal site can be described as a repository array made of a large number of units inside a low permeability layer, called host layer, e.g clay. This clay layer is embedded between layers with higher permeability (Bourgeat et al. [2003]). There is a large number of units, each of them has a small size (10 $\mathrm{m})$ compared with the layer size $(100 \mathrm{~m})$. So a direct numerical simulation of the whole field, based on a microscopic model, is not realistic. The ratio between the width 1 of a single unit and the layer length $\mathrm{L}$ can be considered as a small parameter $\epsilon$ in the detailed microscopic model. The study of the renormalized model behavior, as $\epsilon$ tends to 0 , by means of the homogenization method and boundary layers, gives an asymptotic model which could be used as a global repository model for numerical simulations. According to this rescaling, the units have a height of order $\epsilon^{\beta}, \beta>1$, and are embedded in a layer of thickness $\epsilon$. The leaking of a disposal unit is represented by a hole in 
the periodic computational domain with a given flux on the boundaries. The radioactive pollutant is transported both by the convection due to the water flowing slowly through the rocks (creeping flow) and by the diffusion due to the dilution into the water. The transport of concentration of an underground pollutant is modelled by the advection-diffusion equation (where the advection velocity is assumed to be given) that follows:

$$
\frac{\omega R \partial c}{\partial t}-\nabla \cdot(A \nabla c)+(U \cdot \nabla) c+\lambda \omega R c=q \text { in } \Omega
$$

where: $\Omega$ is the porous medium, $c$ a radioactive component concentration in the water, $\omega$ the medium porosity, $\mathrm{R}$ the retardation coefficient, $U$ Darcy's velocity, $A$ the diffusive term, $\lambda$ the radioactive decay, q the source term.

We normalize the geometric dimensions taking into account the ratio $\epsilon$. The process is then described by the following advection-diffusion type equation:

$$
\begin{array}{rr}
\frac{\omega^{\epsilon} \partial c_{\epsilon}}{\partial t}-\nabla \cdot\left(A_{\epsilon} \nabla c_{\epsilon}\right)+\left(v^{\epsilon} \cdot \nabla\right) c_{\epsilon}+\lambda \omega^{\epsilon} c_{\epsilon}=0 \text { in } \Omega_{\epsilon}^{T} \\
c_{\epsilon}(0, x)=c_{0}(x), \quad x \in \Omega_{\epsilon} \\
n \cdot \sigma=n \cdot\left(A^{\epsilon} \nabla c_{\epsilon}-v^{\epsilon} c_{\epsilon}\right)=\Phi(t) & \text { on } \Gamma_{\epsilon}^{T} \\
c_{\epsilon}=0 & \text { on } S_{1} \\
n \cdot\left(A^{\epsilon} \nabla c_{\epsilon}-v^{\epsilon} c_{\epsilon}\right)=0 & \text { on } S_{2}
\end{array}
$$

where $\Omega_{\epsilon}$ is the adimensionalized domain around the units, $\left.\Omega_{\epsilon}^{T}=\Omega_{\epsilon} \times\right] 0, T[$, $\left.\Gamma_{\epsilon}^{T}=\partial B_{\epsilon} \times\right] 0, T\left[\right.$ where $B_{\epsilon}$ is the set of the units, $S=\partial \Omega_{\epsilon}, S_{1}$ (respect. $S_{2}$ ) represents the bottom (respect. the top) of $S, c_{\epsilon}$ is a radioactive component concentration in the water, $\omega^{\epsilon}$ is the adimensionalized medium porosity, $v^{\epsilon}$ is the adimensionalized convection, $A^{\epsilon}$ the adimensionalized diffusion tensor, $\lambda$ the radioactive decay, $\Phi$ the incoming flux of radioactive element.

It was proved in (Bourgeat et al. [2003]) this $c^{\epsilon}$ exists is unique and has a weak limit $c$. This weak limit $c$ gives the global long time behavior of the process only if the flux $\Phi$ is not too large. On the one hand, we expect some fast oscillations of the solution in the vicinity of the containers and therefore we introduce in that region the fast variable $y=\frac{x}{\epsilon}$. On the other hand, $c_{\epsilon}$ is expected to have the same behavior as the weak limit $c$ without any oscillations far from the units area. These behaviors suggest to use matched asymptotic expansions:

The domain is split in two parts:

- $\left.G_{\epsilon}=\right]-\delta / 2, \delta / 2[\times]-\epsilon \log (1 / \epsilon), \epsilon \log (1 / \epsilon)[$, the inner domain

- $\Omega / \bar{G}_{\epsilon}$, the outer domain.

$\delta$ is defined such that $\partial \Omega \bigcap \partial G_{\epsilon}=\varnothing$

In $G_{\epsilon}$, we look for an asymptotic expansion of $c_{\epsilon}$ such as: 


$$
c_{\epsilon} \simeq c_{\epsilon}^{0}+\epsilon\left(\chi_{\epsilon}^{k}\left(\frac{x}{\epsilon}\right) \frac{\partial c_{\epsilon}^{0}}{\partial x_{k}}+w_{\epsilon}\left(\frac{x}{\epsilon}\right) \Phi-c_{\epsilon}^{0} \rho_{\epsilon}^{k}\left(\frac{x}{\epsilon}\right) v_{k}^{1}\right) \equiv c_{\epsilon}^{1}
$$

where we assume the summation from 1 to 2 over the index $\mathrm{k}$. The function $c_{\epsilon}^{0}$ mimics the behavior of the concentration far from the source. $\chi_{\epsilon}^{k}$ represents the correction on the diffusive term in the near field, $\rho_{\epsilon}^{k}$, the correction on the convective term and $w_{\epsilon}$ the correction on the source. Their behaviors are described by the way of the following type of auxiliary problem:

$$
\left\{\begin{array}{l}
-\nabla \cdot(A \nabla u)=f \text { in } G_{\epsilon} \\
n \cdot(A \nabla u)=g \text { on } \partial M_{\epsilon} \\
u \text { is 1-periodic in } y_{1} \\
\lim _{y_{2} \rightarrow \infty} A \nabla u=r
\end{array}\right.
$$

In order to evaluate the validity of this asymptotic expansion, accurate simulations of these behaviors are needed.

\section{Numerical solutions of the auxiliary problems}

The behavior of the 1-order terms of the homogenization is represented by a diffusive problem on a domain admitting a hole and periodic conditions in the $x$-direction. These problems need accurate discretisation, since they represent the oscillations at the beginning of the leak, and will influence the rest of the simulation. Spectral discretization leads to have structured data-blocks on spectral meshes, then the domain is decomposed into three subdomains, $\Omega_{1}, \Omega_{2}$ and $\Omega_{3}$ in order to take into account the hole. The physical domain size in $x$-direction $[0,1]$ is mapped with a linear mapping to the computational domain $\left(\Omega_{1}\right.$ and $\left.\Omega_{3}\right)$ size in $x$ direction $[0, \pi]$. The decomposition of the computational domain is illustrated in figure 1 . $\Omega_{2}$ has Neumann boundary conditions in the $x$-direction leading to use Chebychev discretisation while $\Omega_{1}$ and $\Omega_{3}$ have periodic boundary conditions in $x$-direction leading to use Fourier discretisation. Thus, meshes between subdomains do not match so spectral mapping techniques are used to represent the solution on both meshes on the artificial interfaces. This accurate representation of the iterate solution on the artificial interfaces generated by the domain decomposition method will allow us to use the Aitken acceleration method developed in (Garbey and Tromeur-Dervout [2002]).

\subsection{Computation in subdomains}

The diffusion tensor $A$ is assumed to depend only on the vertical direction. The solution in subdomains $\Omega_{1}$ and $\Omega_{3}$ is computed on an extended subdomain in order to avoid the 0-mode singularity (because the problems are defined up to a constant). Thus, we compute $\tilde{u}:[0,2 \Pi] \times[-1,1] \rightarrow R$, an odd periodic 

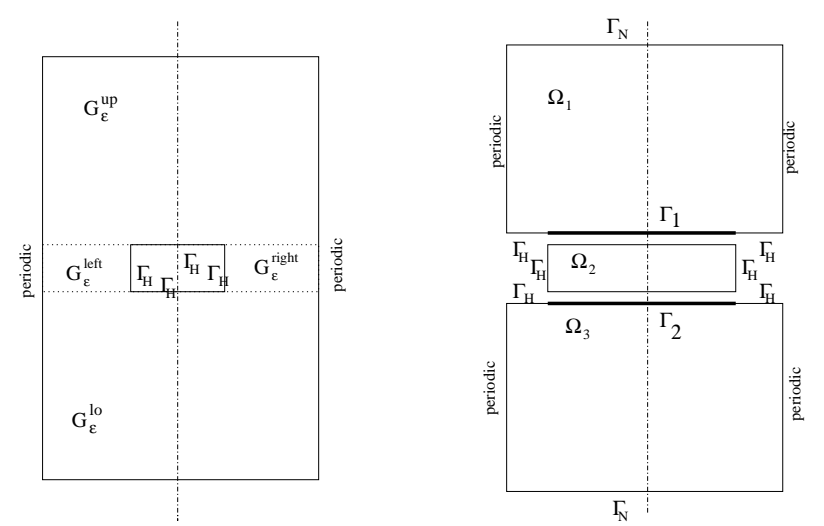

Fig. 1. Decomposition of the computational domain: $\Omega_{1}$ (resp. $\Omega_{3}$ ) corresponds to the upper (resp. lower) part of the domain, $\Omega_{2}$ corresponds to $G_{\epsilon}^{\text {right }} \cup G_{\epsilon}^{\text {left }}$

function, which is equal to $u$, solution of problem (8) on $[0, \Pi] \times[-1,1]$. The extended solution is then, using Chebychev discretisation in $y$-direction, approximated by: $P_{M} \tilde{u}\left(x_{i}, y_{j}\right)=\sum_{0 \leq k \leq M} \sum_{0 \leq l<N} \hat{\tilde{u}}_{k, l} T_{l}\left(y_{j}\right) \sin \left(k x_{i}\right)$, where $x_{i}=\frac{2 i \Pi}{M}, \quad i=0, \ldots, M$, and $y_{j}=\cos \left(\frac{j \Pi}{N}\right), \quad j=0, \ldots, N$. So we obtain for subdomain $\Omega_{1}$ :

$$
\begin{gathered}
\nabla \cdot(A(y) \nabla \tilde{u})=\sum_{0 \leq k \leq M}\left(\frac{\partial}{\partial y}\left(A_{2}(y)\left(\frac{\partial}{\partial y}\right)\right)-k^{2} A_{1}(y)\right) \hat{u}_{k}(y) \sin (k x) \text { in } \Omega_{1}, \\
A(y) \nabla \tilde{u}=\sum_{0 \leq k \leq M} A_{2}(y) \frac{\partial}{\partial y}\left(\hat{u}_{k}(y)\right) \sin (k x) \text { on } \Gamma_{1},
\end{gathered}
$$

where $\hat{u}_{k}(y)=\sum_{0 \leq j \leq N} \hat{\tilde{u}}_{k, j} T_{j}(y)$. Due to the linearity, the solution $P_{M} \tilde{u}$ can be decoupled according to the directions. Thus, the subdomain problem is decoupled in M mode-problems of size $(N+1) \times(N+1)$ leading to a well suited situation for the parallelization.

Some difficulties appear due to the boundary condition on $G_{1}=\{y=$ $\left.y_{N}=-1\right\}$, coming from the decomposition domain:

$$
\left\{\begin{array}{l}
\frac{\partial u_{1}(x, y)}{\partial y}=\frac{\partial u_{2}(x, y)}{\partial y}, \quad \text { on } \Gamma_{1}=\left[x_{1}, x_{2}\right] \times\{-1\} \\
n \cdot\left(A \nabla u_{1}\right)=g(x, y), \text { on } G_{1} \backslash \Gamma_{1}
\end{array}\right.
$$

The boundary condition on $G_{1}$ is singular. In order to use a discrete Fourier transform, smoothing methods have to be applied. First, a $C^{2}$ Hermite interpolation based on two points is computed in the vicinity of $x_{1}$ and $x_{2}$ like in Garbey and Tromeur-Dervout [1998]. Then the "raised cosinus filter" (Gottlieb and Shu [1996]) is applied on the modes of the traces on the extended boundary condition including $G_{1}$, (in the same way as $\tilde{u}$ ) in order to minimize the Gibbs phenomenon. The third domain $\Omega_{3}$ is treated in the same way. 
On the second subdomain $\Omega_{2}$, a Chebychev-Chebychev discretisation is used in both directions. Now, the pseudospectral discretisation of the differential operator leads to solve a $\left(\left(N_{1} N_{2}\right) \times\left(N_{1} N_{2}\right)\right)$ full but time independent linear system with a PLU factorization. Parallel ADI techniques are also under implementation to save time.

\subsection{Methodology on Neumann-Dirichlet Domain Decomposition and Aitken Acceleration method}

The Neumann-Dirichlet domain decomposition method leads to solve for the auxiliary problems:

$$
\begin{array}{rc}
\nabla \cdot\left(A \nabla u_{1}^{n+1 / 2}\right)=f & \text { in } \Omega_{1} \\
\frac{\partial}{\partial n} u_{1}^{n+1 / 2}=\frac{\partial}{\partial n} u_{2}^{n} & \text { on } \Gamma_{1} \\
\nabla \cdot\left(A \nabla u_{3}^{n+1 / 2}\right)=f & \text { in } \Omega_{3} \\
\frac{\partial}{\partial n} u_{3}^{n+1 / 2}=\frac{\partial}{\partial n} u_{2}^{n} & \text { on } \Gamma_{2} \\
\nabla \cdot\left(A \nabla u_{2}^{n+1}\right)=f & \text { in } \Omega_{2} \\
u_{2}^{n+1}=u_{1}^{n+1 / 2} & \text { on } \Gamma_{1} \\
u_{2}^{n+1}=u_{3}^{n+1 / 2} & \text { on } \Gamma_{2}
\end{array}
$$

Using Aitken acceleration method for the Schwarz DDM based on the linear convergence or divergence of the iterative method (Garbey and TromeurDervout [2002]), the convergence of the solution on the artificial interface can be speed up in few iterations. The linear convergence can only be obtained for the iterated solution Fourier modes on the artificial interfaces. The solution $u$ on $\Gamma_{1}$ and $\Gamma_{2}$ on the Chebychev grid being not periodic, $\left.u\right|_{\Gamma_{1}}$ is left-extended on $[0, \pi]$ with a fifth-degree Hermite interpolation. Then we seek an odd solution on the extended domain $[0,2 \pi]$.

The distance between the artificial interfaces $\Gamma_{1}$ and $\Gamma_{2}$ is small. Thus these interfaces are coupled for low modes. Let us consider the sequence $\hat{u}_{k}^{n}=$ $\left(\hat{u}_{k, 1 \mid \Gamma_{1}}^{n}, \hat{u}_{k, 3 \mid \Gamma_{2}}^{n}\right)^{t} k=0, \cdots, M$. The operator $T_{k}$,

$$
\left(\hat{u}_{k}^{n}-\hat{U}_{k}^{\infty}\right) \rightarrow\left(\hat{u}_{k}^{n+1}-\hat{U}_{k}^{\infty}\right)
$$

where $U_{k}^{\infty}$ is the $\mathrm{k}$ mode of the exact solution, is linear. As long as the artificial interfaces are coupled, the matrix $P_{k}, k=0, \cdots, M$ associated to the operator $T_{k}$ is full.

From (16), we have:

$$
\hat{u}_{k}^{i+2}-\hat{u}_{k}^{i+1}=P_{k}\left(\hat{u}_{k}^{i+1}-\hat{u}_{k}^{i}\right), i=n-1, n ; k=0, \cdots, M .
$$

We notice that, for each mode, only three iterations are needed to determine the coefficients of the matrix $P_{k}$ with( 17$)$. If the operator $I d-P_{k}$ is not singular, then the Aitken acceleration will be written as follows for each mode: 


$$
\hat{U}_{k}^{\infty}=\left(I d-P_{k}\right)^{-1}\left(\hat{u}_{k}^{n+1}-P_{k} \hat{u}_{k}^{n}\right), k=0, \cdots, M .
$$

Finally, a backward Fourier transform on these $\hat{U}_{k}^{\infty}$ gives the solution in the physical space on the artificial interfaces. The extension of the solution on the artificial interfaces is a $C^{2}$ function and it is enough to get a three order rate for the discretisation error of our approximation.

Remark 1: as the smoothing procedure introduces some non linearities, more than one acceleration has to be applied on the Neumann-Dirichlet algorithm. Remark 2: the linear behavior of the mode error for this Schwarz DDM with smoothing procedures is not obtained directly (because of the Gibbs phenomenon), so that the Aitken speed-up can be applied only after some iterations.

\section{Numerical Results}

We first checked the accuracy of the method on an analytical solution $u$ : $(x, y) \rightarrow \sin (x) \sin (y)$ of an elliptical problem defined on the computational domain $\Omega$. Figures 2 and 3 show the Aitken acceleration (at iteration 20) effect on usual Schwarz convergence (the error is the $\|\cdot\|_{\infty}$ of the difference between two successive iterations for each mode).

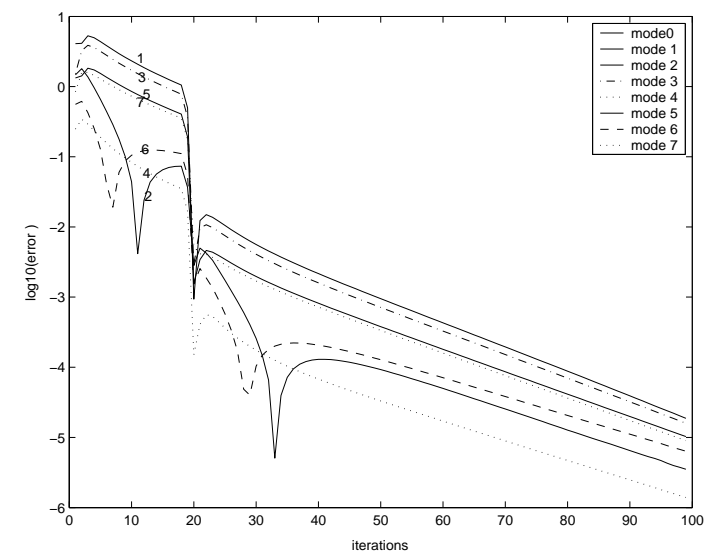

Fig. 2. Aitken acceleration of the mode-error (after 16 iterations) for the analytical problem

The error in the table 1 shows the $\|\cdot\|_{\infty}$ of the difference between the exact trace and the computed solution on artificial interfaces. The method is of order-2.5 of consistency instead of an expected order-3. Nevertheless, the acceleration of the convergence thanks to the Aitken speed-up is satisfactory (30 iterations instead of 100) for the same accuracy. 


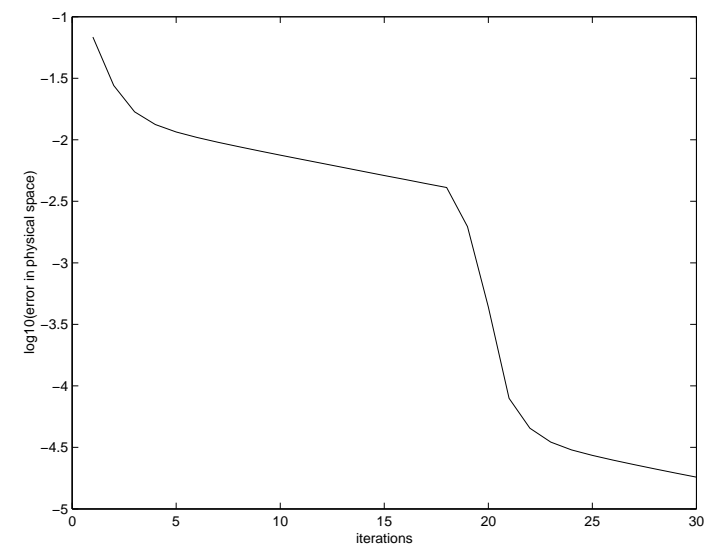

Fig. 3. Aitken acceleration of the error in the physical space (after 16 iterations) for the analytical problem

Table 1. Precision and velocity of the algorithm

\begin{tabular}{|c|c|c|c|c|c|}
\hline Accel./ & modes nb. & y-discr. & Precision & iterations nb. & Time(s) \\
\hline No acceleration & 40 & 32 & 0.05 & 100 & 22,77 \\
\hline No acceleration & 80 & 32 & 1.e-4 & 100 & 27,07 \\
\hline No acceleration & 178 & 32 & 7.e-5 & 100 & 41,64 \\
\hline No acceleration & 256 & 32 & 4.e-5 & 100 & 58,82 \\
\hline Acceleration & 178 & 32 & 7.e-5 & 30 & 13,44 \\
\hline
\end{tabular}

If we now apply our methodology on one of the auxiliary problems, for instance the corrector $\rho_{\epsilon}^{k}$ on the convective term, Fig. 4 shows the isolines of the computed solution of the problem (19), for $\mathrm{k}=2$, obtained with the present methodology in 12 iterations.

We recall that $\rho_{\epsilon}^{k}$ follows the equation:

$$
\left\{\begin{array}{l}
-\nabla \cdot\left(A \nabla \rho_{\epsilon}^{k}\right)=0 \text { in } G_{\epsilon} \\
n \cdot\left(A \nabla \rho_{\epsilon}^{k}+e_{k}\right)=0 \text { on } \partial M_{\epsilon} \\
\rho_{\epsilon}^{k} \text { is 1-periodic in } y_{1} \\
\lim _{y_{2} \rightarrow \infty} \nabla \rho_{\epsilon}^{k}=0
\end{array}\right.
$$

\section{Conclusion}

Our aim was to develop a methodology adapted to a physical problem, which cannot be easily simulated directly. The field decomposition splits the solution into regular problems according to the physical situation. In order to speed up 


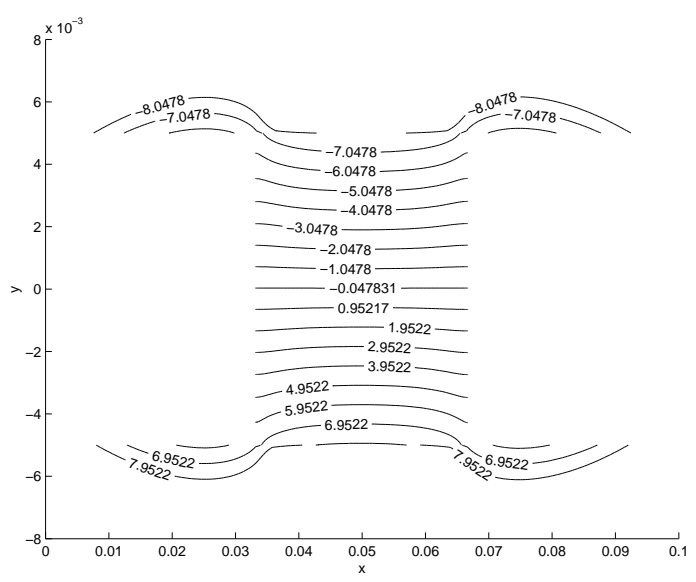

Fig. 4. $\rho_{k}, \mathrm{k}=2$, isolines in the vicinity of the artificial interfaces for problem (19)

this Schwarz method by Aitken process, spectral methods are helping to obtain an accurate representation of the Neumann-Dirichlet algorithm iterations on a non-matching grid. This methodology has been applied on a problem with an analytical solution and clearly accelerates the speed of convergence of the Schwarz method. The same methodology was also applied successfully on all the auxiliary problems of the 1-order of the asymptotic expansion. The computation of the 0 order term of the model is currently under development (the Aitken-Schwarz method is applied on mixed finite element / spectral element systems).

\section{References}

A. Bourgeat, O. Gipouloux, and M. Marusic-Paloka. Modelling of an underground waste disposal site by upscaling. In Math. Meth. in Appl. Sci., pages 152-158. 2003.

M. Garbey and D. Tromeur-Dervout. A new parallel solver for non periodic incompressible navier stockes equation with fourier basis: Application to frontal polymerisation. In J. Comp. Phys.145, pages 316-331. 1998.

M. Garbey and D. Tromeur-Dervout. On some aitken like acceleration of the schwarz method. In Int.J. For Numerical Methods In Fluids 40(12), pages 1493-1513. 2002.

D. Gottlieb and C.-W. Shu. On the gibbs phenomenom and its resolution. In SIAM J. Sci. Comput. 39(4), pages 644-668. 1996. 